\title{
Erratum: Theory of a Polarized Fermi Liquid in Two Dimensions: Spin Diffusion*
}

\author{
K. Miyake $\dagger$ and W. J. Mullin $\ddagger$ \\ Laboratory for Low Temperature Physics, University of Massachusetts, Amherst, Massachusetts, \\ and School of Mathematical and Physical Sciences, University of Sussex, Falmer, Brighton, \\ England
}

(Received October 22, 1984)

Figure 2 contains three errors: $\mathbf{p}_{2}$ should be replaced by $\mathbf{p}$ in two places and $\sum_{\mathbf{p}}$ should be inserted in the last expression as shown in the accompanying corrected figure.

$$
\int d \epsilon_{2} \sum_{\overrightarrow{\mathrm{a}}_{2}} \int d \epsilon \sum_{\overrightarrow{\mathrm{p}}}
$$

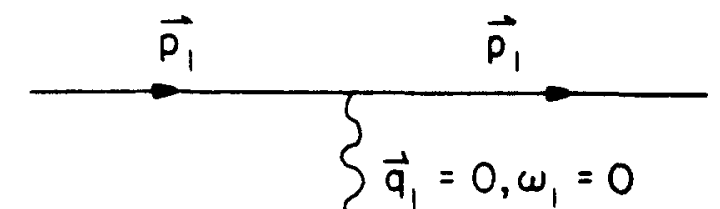

*This paper appeared in J. Low Temp. Phys. 56, 499 (1984).

$\dagger$ Permanent address: Department of Physics, Nagoya University, Nagoya, Japan.

$\ddagger$ Permanent address: Laboratory for Low Temperature Physics, Hasbrouck Laboratory, University of Massachusetts, Amherst, Massachusetts. 